\title{
Effective PocketLab Sensor Use in an Engineering Dynamics Course
}

\section{Dr. Timothy Aaron Wood, The Citadel}

Timothy A Wood is an Assistant Professor of Civil and Environmental Engineering at The Citadel. He acquired a Bachelor's in Engineering Physics Summa Cum Laude with Honors followed by Civil Engineering Master's and Doctoral degrees from Texas Tech University. His technical research focuses on the intersection of soil-structure interaction and structural/geotechnical data. He encourages students pushing them toward self-directed learning through reading, and inspiring enthusiasm for the fields of structural and geotechnical engineering. Dr. Wood aims to recover the benefits of classical-model, literature-based learning in civil engineering education. 


\title{
Effective PocketLab Sensor Use in an Engineering Dynamics Course
}

\begin{abstract}
PocketLab sensors effectively contribute to the engineering dynamics classroom by illustrating fundamental kinematic relationships, allowing students to measure kinematic properties, confirming theoretical predictions, and calculating reactions. Though developed for K-12 education, PocketLab sensors are small, relatively inexpensive devices that allow users to record acceleration, range, and angular velocity through a Bluetooth connection to a smart phone, tablet, or laptop. The real-time presentation of data illustrates the relationship between acceleration, velocity, and position while also allowing students to calculate related reactions and forces driving motion. A collection of example problems and demos involving the PocketLab sensor illustrate various dynamics concepts. Student survey results indicate the usefulness of in-class demonstrations with the PocketLabs for reinforcing dynamics concepts.
\end{abstract}

\section{Keywords}

In-class Demonstration, Engineering Mechanics, Student Perspectives

\section{Introduction}

The engineering dynamics course is an often-challenging hurdle for civil and mechanical engineering students. Students often struggle to apply vector mathematics to difficult to visualize displacements, velocities, and accelerations. Studies and experience have indicated that physical models in the classroom can be helpful for students; yet, connecting numbers to motion can be challenging [1]. The benefits of physical experiments in the classroom that bring the dynamics concepts and equations to life are well documented [2]. The experiments that actually measure acceleration and other kinematic properties can be very effective. To this end, engineering educators have developed experiments that take advantage of the accelerometers in smart phones [3]-[6]. One educator has incorporated motion tracking software for analysis of videos [7]. Yet others have built measurement solutions from component accelerometers, kit computers, and gyroscopes [8]-[11]. Easy to use and low cost PocketLab sensors continue in this development of instrumentation and effectively contribute to the engineering dynamics classroom by illustrating fundamental kinematic relationships, allowing students to measure kinematic properties, confirming theoretical predictions, and calculating reactions. 


\section{Description}

PocketLab sensors, developed for K-12 education, provide an easy-to-use way to measure physically observable motion with real time numbers. The PocketLab Voyager device measures 1.5in. x 1.5in. $x$ 0.6in. and has a multitude of onboard sensors, most notably for dynamics: an infrared range finder, accelerometer, and gyroscope [12]. A wireless Bluetooth connection to a laptop or tablet puts these sensors and data at the student's fingertips in real-time at up to $50 \mathrm{~Hz}$. The PocketLab app on iOS/padOS/Android smoothly connects and gives the user easy access to data collection, digital read out, or real-time graphs of up to three sensor channels. The accelerometer measures accelerations and gravitation acceleration in three-dimensions. Dimensional directions are indicated using the PocketLab logo, a right-handed Cartesian coordinate system (see Figure 1). A range of data collection rates are available, and the on-screen view can show graphs alone or real-time video through the tablet or computer's camera. Recorded data is accessible as a CSV file or a real-time plot of the data over a video. After recording, the graph explorer allows for easy reading of individual data points at specific times. The net result is a seamless integration of physical testing, data collection and data processing. The PocketLab system is streamlined enough to be used real-time in a 50-minute college lecture. The PocketLab company also sells various three-dimensional printable models for carts and pendulums, as well as, additional add-on sensors. The PocketLab Voyager can also be set in data logging mode and store up to 30,000 data points. All this power and functionality comes in a device that costs less than $\$ 150$.

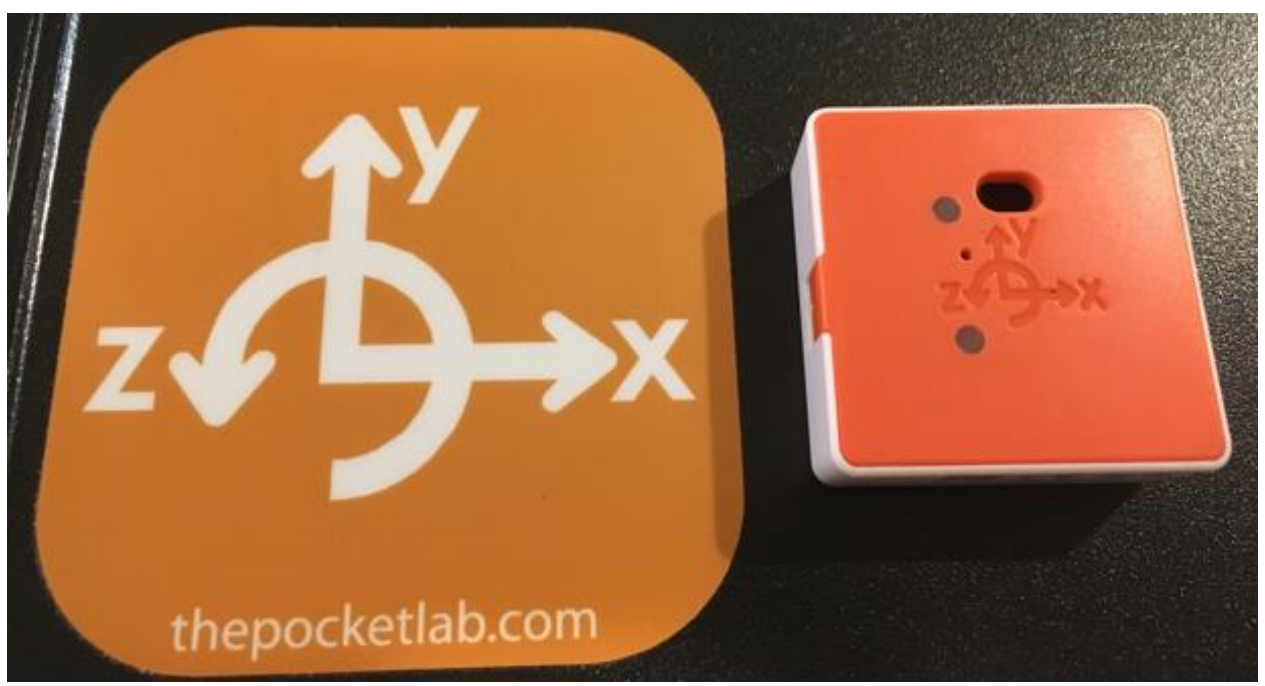

Figure 1. PocketLab logo and Cartesian coordinate sensor directions (actual size) 


\section{Applications}

The author has developed PocketLab demonstrations for most subareas of dynamics. These demonstrations use the PocketLab device to measure some aspect of the motion setting up the calculation of other aspects of the motion as an example problem on the board. Though each experiment has pre-recorded data incorporated into a PowerPoint presentation, most experiments can be replicated in a timely manner in the classroom to better help student understanding. Many additional experiments appropriate for all educational levels are available through the Pocket Labs Educator website [13].

\section{Particle Kinematics}

In the area of particle kinematics, the PocketLab Velocity Lab app is very helpful. The PocketLab is attached to a wheel, the app requests the diameter of the wheel, the PocketLab gyroscope sensor provides data, and the Velocity Lab app translates the data into position, velocity and acceleration as seen in Figure 2. The professor can then discuss the motion real-time as the video plays.
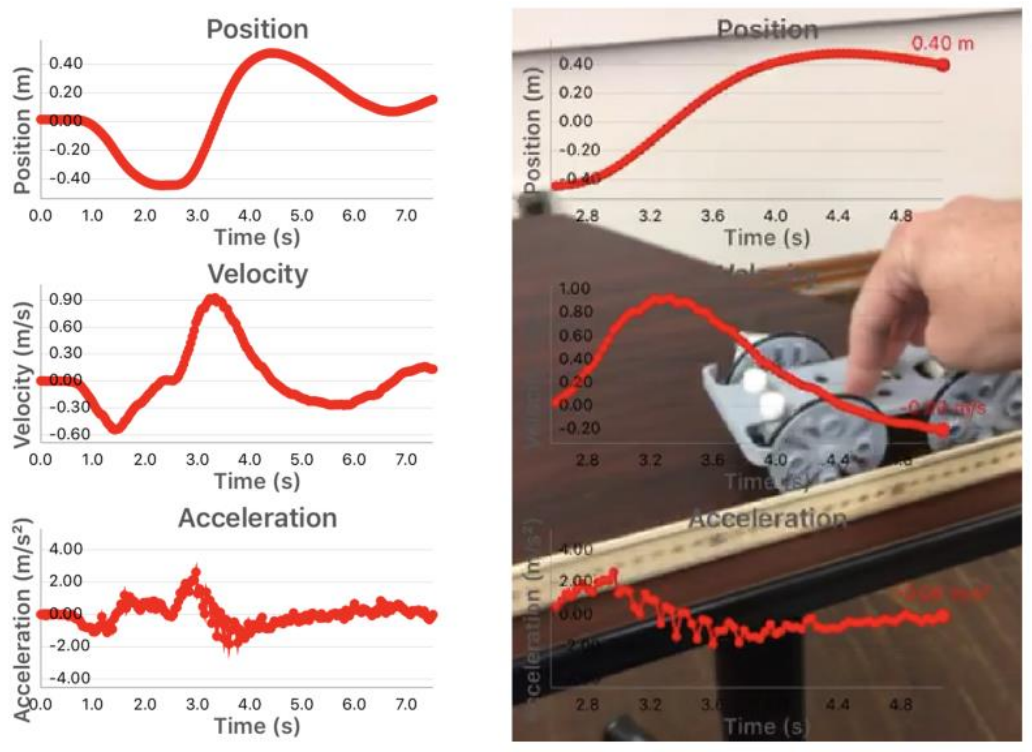

- Rectilinear Motion - Kinematic Properties

- Time, $t$

-Position, $s$ - Velocity, $v$ -Acceleration, $a$

Figure 2. Rectilinear Particle Motion using the Velocity Lab app 
The standard PocketLab application can be used in the Scalar Acceleration mode to explore constant acceleration. The PocketLab is placed in a protective case, thrown in the air, and allowed to hit the ground. The acceleration data shows the upward acceleration while in the hand, the constant acceleration while moving up and back down, and the sharp accelerations of the sensor bouncing on the ground. Figure 3 shows both a video still and a data point highlighted in the data exploration mode. Given this data, the students can measure time of flight (from acceleration jump to acceleration jump) and use constant acceleration equations to calculate initial and final velocities as well as the peak height of the flight.
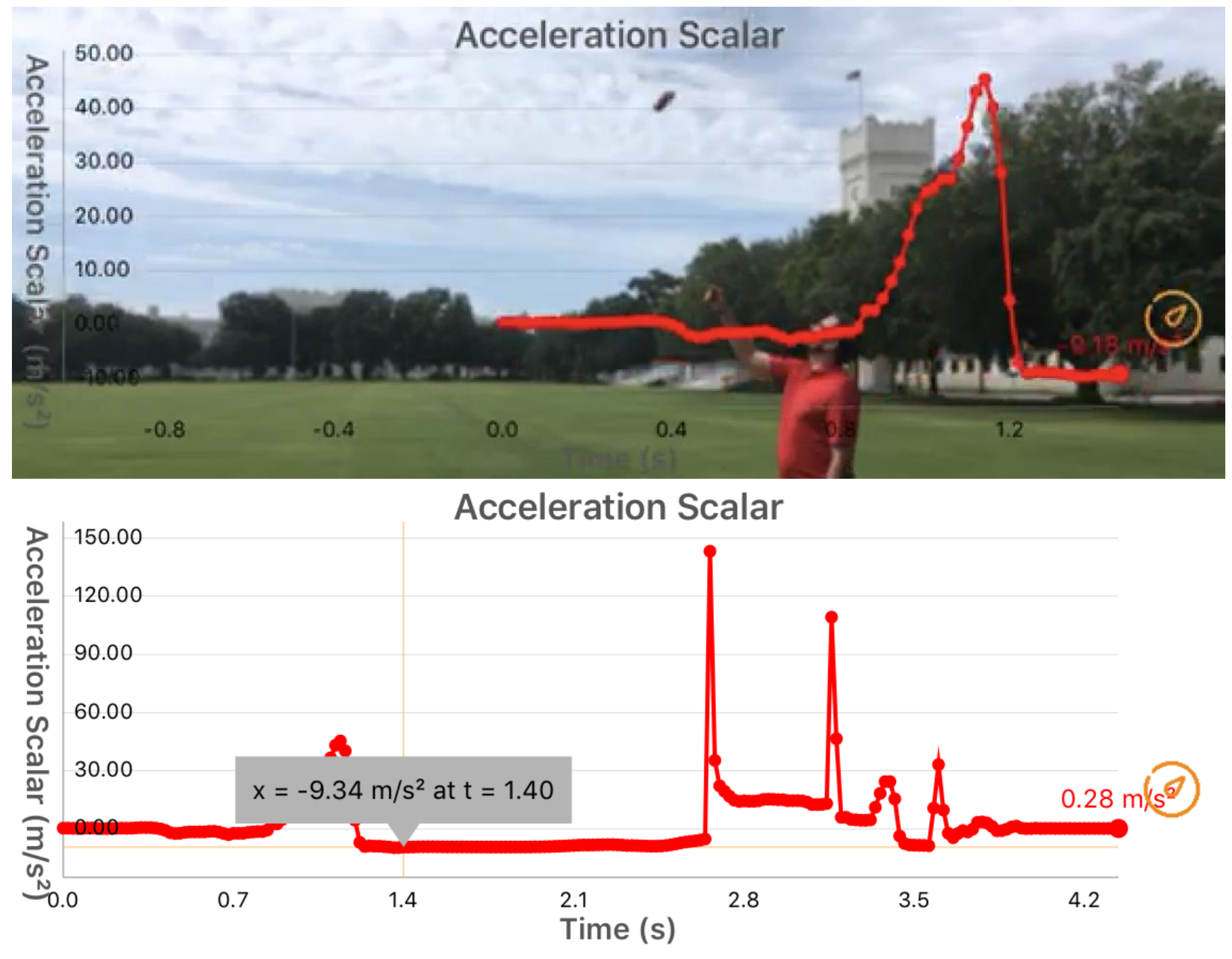

Figure 3. PocketLab scalar acceleration data of the sensor thrown in the air 
Curvilinear motion can be illustrated by using the PocketLab as a bob on a pendulum. In this case the accelerometer $\mathrm{x}$-axis points in the tangential direction, and the accelerometer $\mathrm{y}$-axis points in the normal direction. The accelerometer always measures gravity, so the acceleration due to gravity must be removed from the y-component acceleration at the bottom of the swing. The data illustrates the reasonableness of the theoretical accelerations at the bottom of the swing as seen in Figure 4. The use of PocketLabs in the class to explore various aspects of particle kinematics is very helpful for the students.
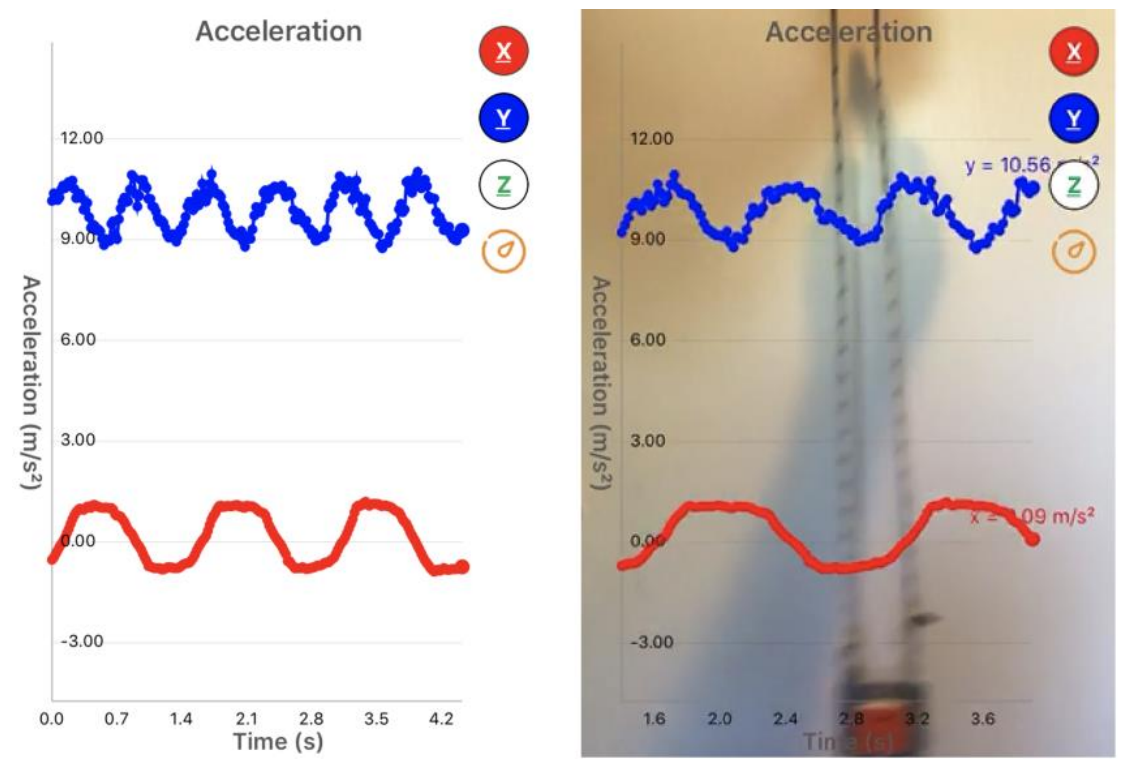

-Tangential: $x$-axis

-At bottom of swing, $a_{t}=0$

-Normal: y-axis

- At bottom of swing,

$a_{n}=g+\frac{v^{2}}{\rho}$

Figure 4. Path coordinate system curvilinear motion illustrated in PocketLab pendulum 


\section{Particle Kinetics}

When exploring particle kinetics, the PocketLab can be used to measure the position, velocity, and/or acceleration and then calculations can estimate force acting on the object. In the demo shown in Figure 5, the PocketLab is attached to a block and the range finder is used to provide position and velocity as the block slides down a ramp. Students can see that measured data can be noisy, but the rangefinder velocity plot can provide the slope of velocity, i.e. the acceleration. From this data, students use the constant acceleration experienced by the block and calculate the coefficient of kinetic friction between the block and the ramp.
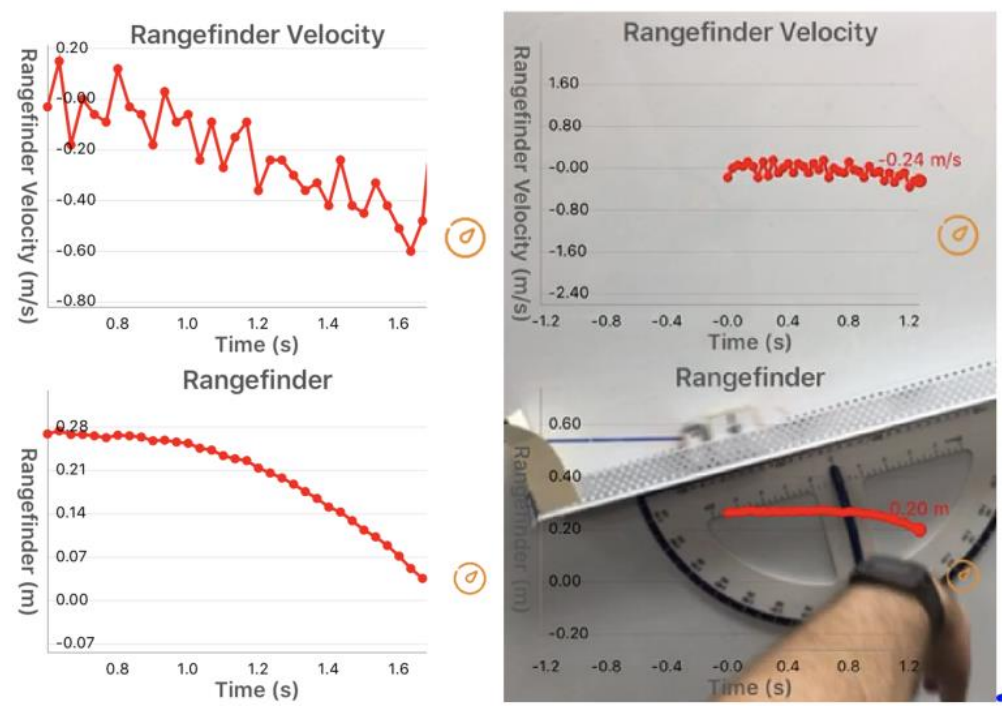

\section{Calculate the coefficient of kinetic friction $m=75 \mathrm{~g}$}

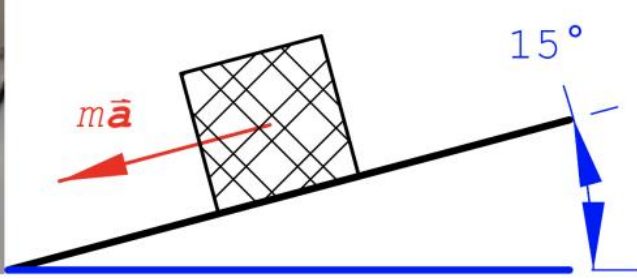

Figure 5. PocketLab rangefinder used to calculate coefficient of friction. 
Knowledge of the coefficient of friction and the weight of the block means that range finder data can also be used to estimate forces and reactions in moving systems. Figure 6 shows a sliding block and pulley system. Using the collected data, students can estimate the tension in the rope using the equations of motion.

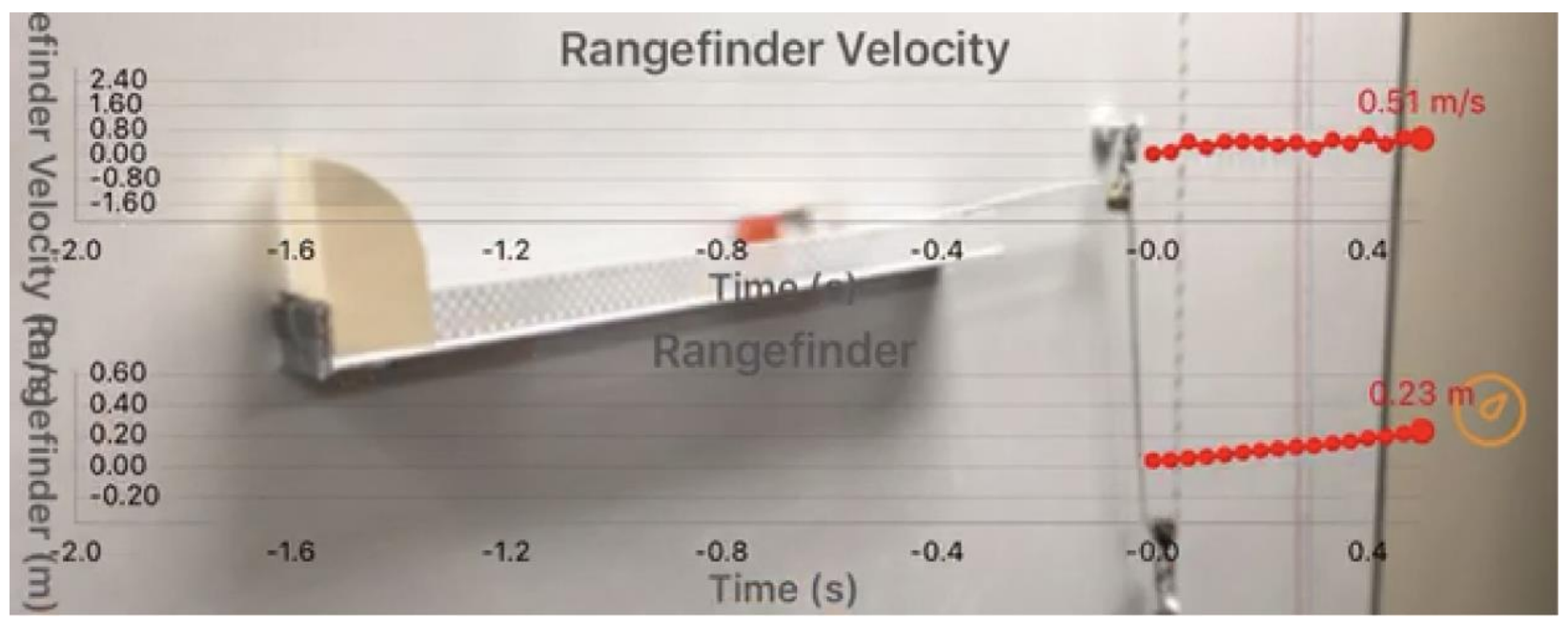

Figure 6. PocketLab rangefinder used to estimate tension in pulley system during motion.

The same data from the pulley system can be incorporated into a work-energy problem. Figure 7 sets up a problem to solve for the speed of the blocks at a given time using work-energy equations.
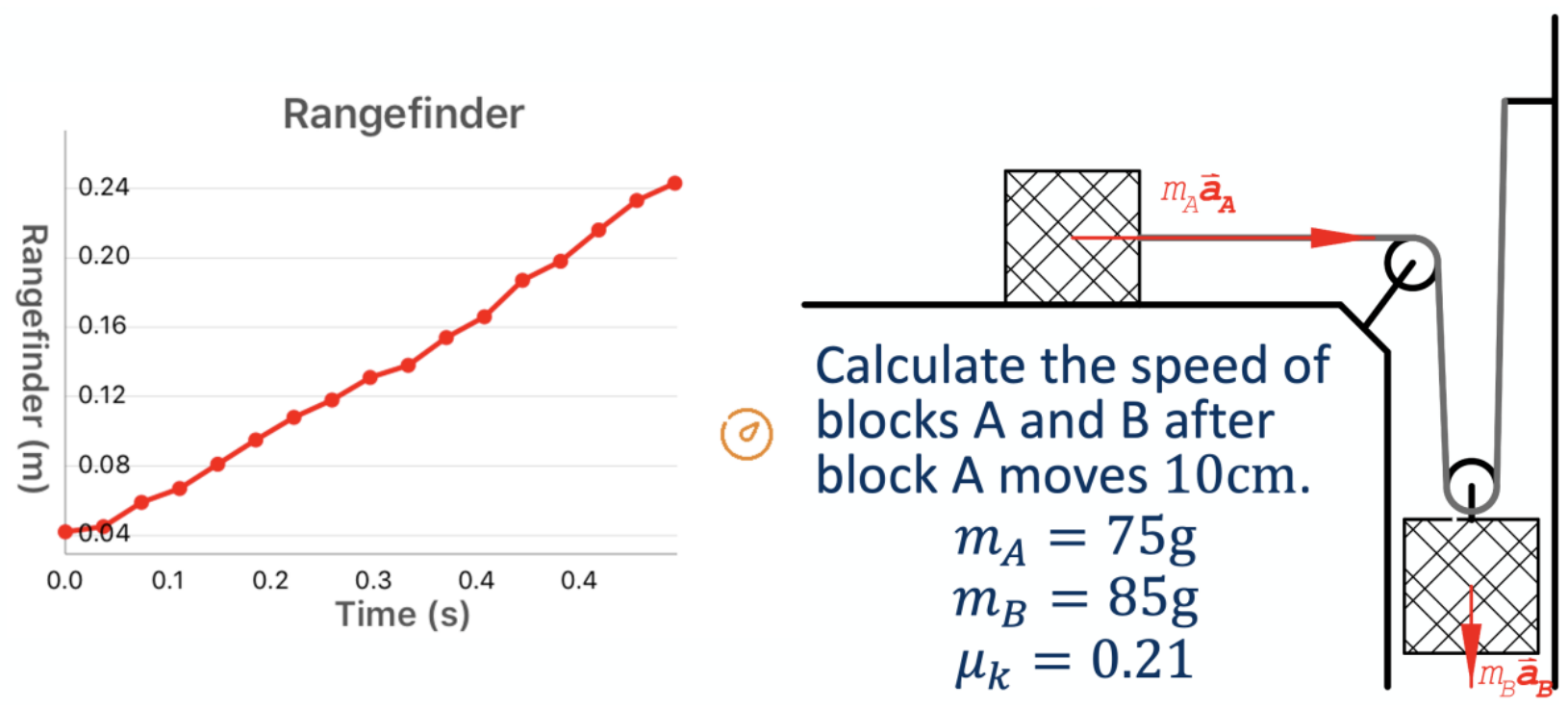

Figure 7. PocketLab rangefinder used to provide data for a work-energy problem. 


\section{Rigid Body Kinematics}

PocketLabs also make it possible to explore rigid body kinematics. In the demonstration shown in Figure 8, the PocketLab Velocity Lab provides all the kinematic properties for the axle on a cart. This data is used to calculate the velocity and acceleration of points on the wheel as well as the acceleration of an action figure riding a board on top of the wheel. Real data makes the example more interactive.
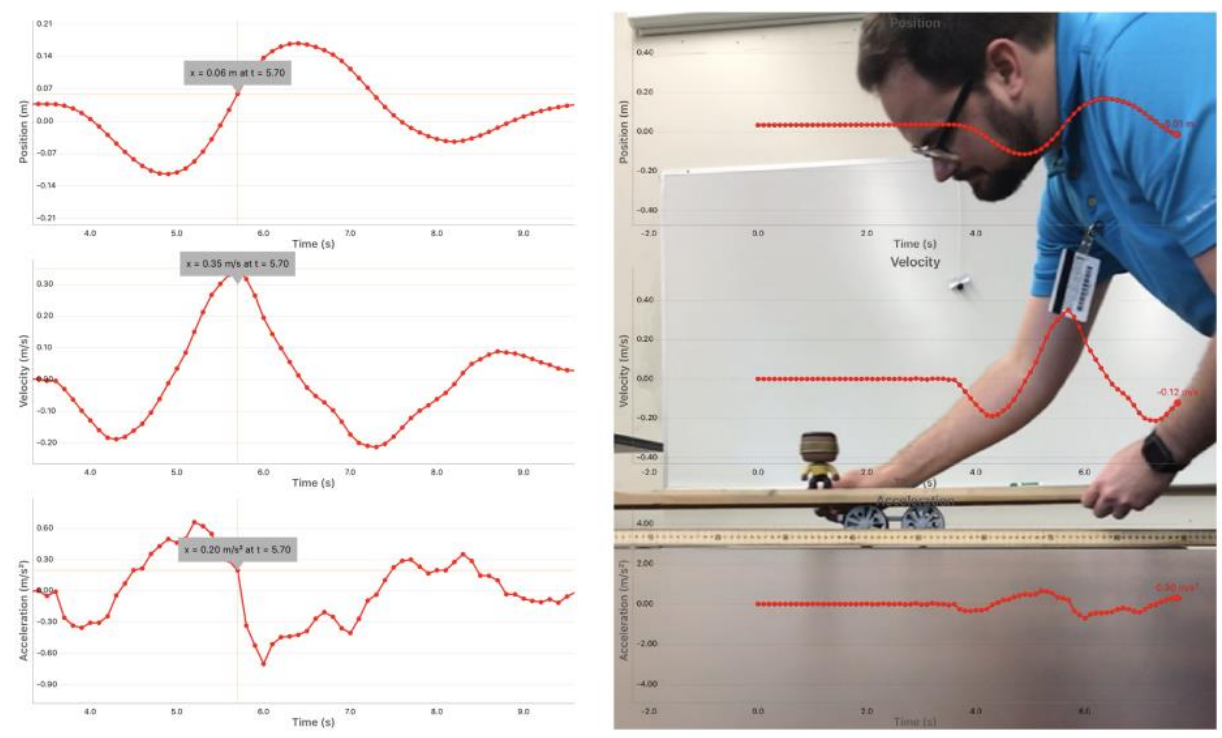

Calculate LCdr La Forge's speed, angular velocity, and the velocity of the far right edge of the wheel.

Figure 8. PocketLab Velocity Lab generating kinematic data for the wheel axle for a rigid body kinematic problem.

\section{Rigid Body Kinetics}

Rigid body kinetics can also be explored using the PocketLab data. In one in-class demo, students participate in office chair racing. A PocketLab sensor is used to measure the deceleration of a coasting chair. From the measurement of the inertial terms at the center of mass, students then calculate the equivalent rolling coefficient of friction for the chair as well as the weight distribution between the front and back legs of the chair. Students seem to enjoy getting to race chairs across the classroom while exploring the kinetic equations of motion that let them calculate friction and reaction forces from the acceleration data.

Rigid body work-energy can also be explored using the PocketLab. The PocketLab measures the motion of the center of a yard stick pendulum. Though the accelerations can be measured (same as in the particle equilibrium example) for the rigid yard stick, the PocketLab gyroscope provides angular velocity about the z-axis. With a measured angular velocity, students can compare the measured velocity to the theoretical velocity from the work-energy equation for the problem. 


\section{Student Perspectives}

Students seem to respond well to examples that include a PocketLab component. Figure 9 show 145 student perspectives from six sections across five semesters on 3 statements about PocketLabs: (1) "PocketLab demonstrations have increased my active involvement in class." (2) "PocketLabs have been beneficial to my learning." And (3) "I hope more professors will use sensors like PocketLabs." Over 95\% of students felt that PocketLabs contributed positively to their classroom involvement and learning. Figure 10 show that nearly half of the students felt that PocketLab-powered demonstrations were the most instructive form of in-class example. The tangible way that observed motion was captured as data may have helped the students in the development of their mental model. Student seem generally to like the PocketLab technology implemented in the classroom.

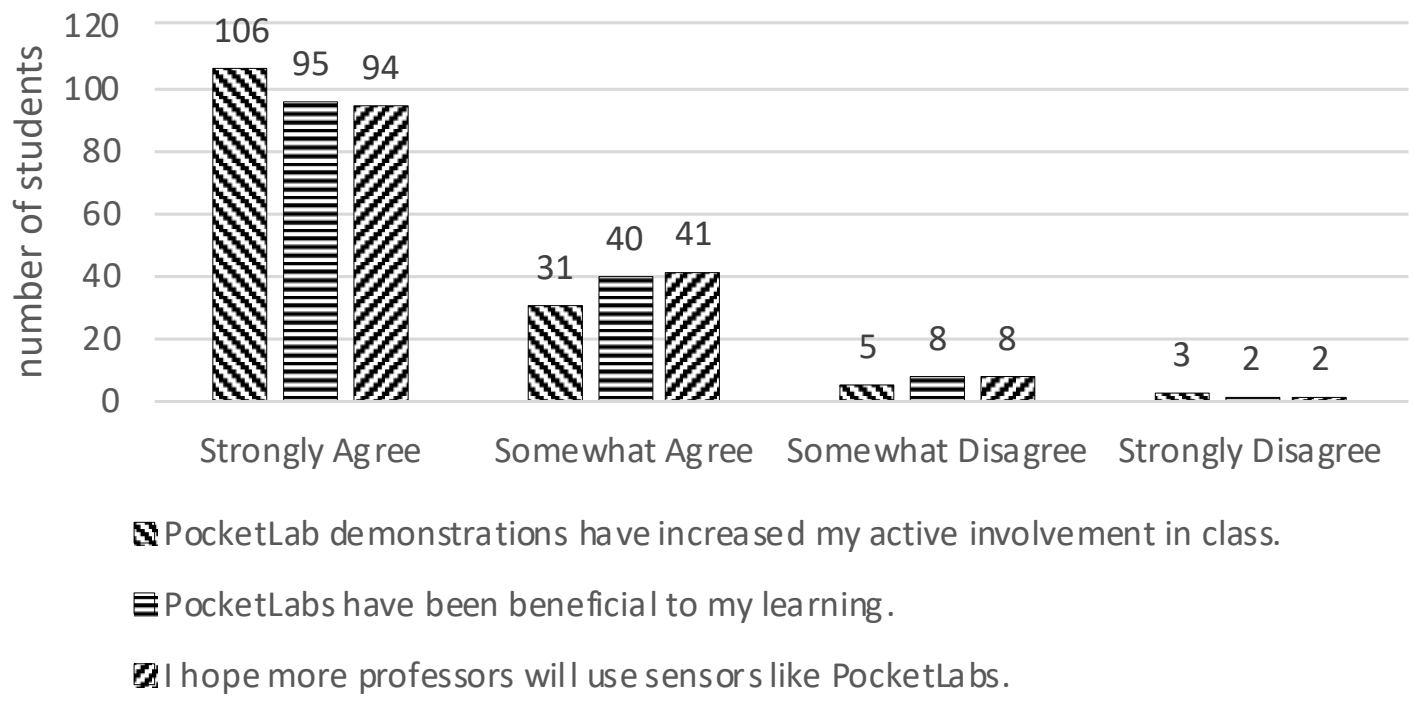

Figure 9. Student perspectives on PocketLabs.

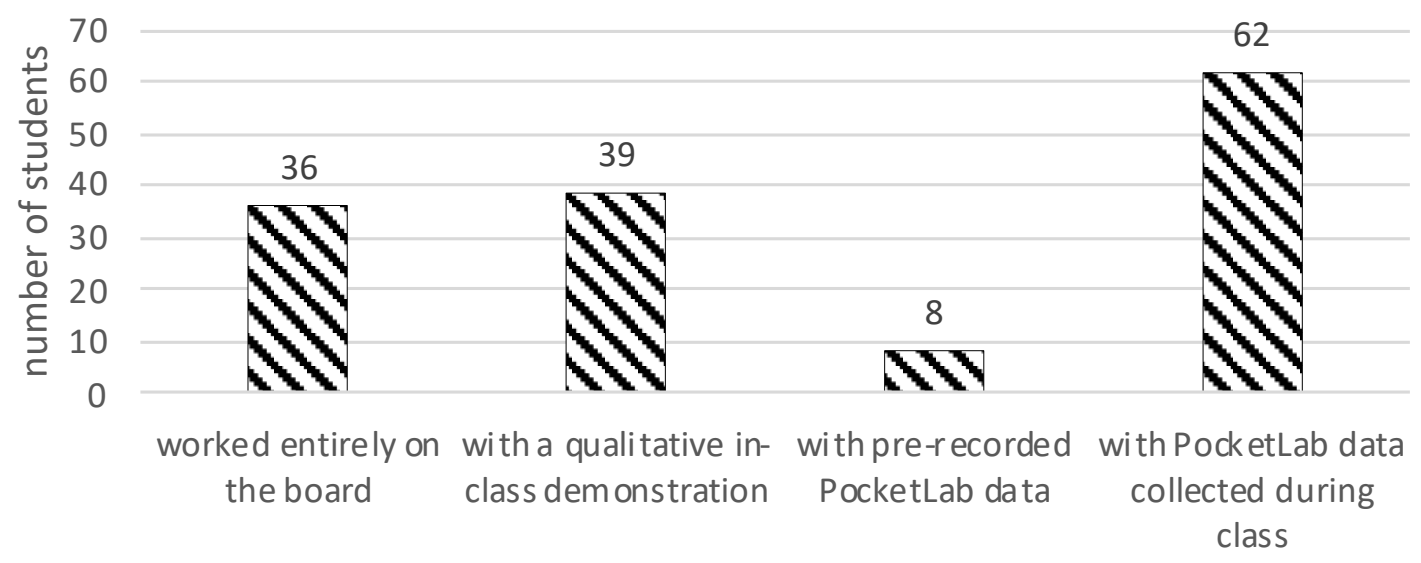

Figure 10. Student responses to the prompt, "My understanding increases most during those inclass examples..." 
Though student response to the PocketLabs was positive, the PocketLabs did very little to improve overall student performance in the class. Considering students taking dynamics with the author, the average Dynamics GPA for 129 students across six sections in four semesters without the PocketLabs demonstrations was exactly the same as the average GPA for 123 students across four semesters with the PocketLabs. Students may have felt that PocketLabs improved the classroom experience, but student performance may be based on more than just the classroom experience, i.e. engagement with homework, study habits, and exam preparation.

\section{Conclusion}

PocketLabs contribute to student understanding in dynamics. The ability to measure and explore accelerations, angular velocities, and position in real-time with relative ease makes them a perfect in-class demonstration. The system is easy to use in class, and the students seem to engage better than with traditional in-class examples. PocketLabs promises to be a powerful tool for teaching dynamics.

\section{References}

[1] A. C. Estes, R. W. Welch, and S. J. Ressler, "The ExCEEd Teaching Model," Journal of Professional Issues in Engineering Education and Practice, vol. 131, no. 4, pp. 218-222, Oct. 2005, doi: 10.1061/(ASCE)1052-3928(2005)131:4(218).

[2] A. A. Ferri and B. H. Ferri, "Blended Learning in a Rigid-Body Dynamics Course Using OnLine Lectures and Hands-On Experiments," presented at the 2016 ASEE Annual Conference \& Exposition, Jun. 2016, Accessed: Mar. 17, 2020. [Online]. Available: https://peer.asee.org/blended-learning-in-a-rigid-body-dynamics-course-using-on-linelectures-and-hands-on-experiments.

[3] D. O'Connor, "Investigations into Engineering Dynamics Theory: A Student-Led Project to Utilize Smartphone Technology," presented at the 2017 ASEE Annual Conference \& Exposition, Jun. 2017, Accessed: Mar. 17, 2020. [Online]. Available: https://peer.asee.org/investigations-into-engineering-dynamics-theory-a-student-led-projectto-utilize-smartphone-technology.

[4] C. D. Facciolo and A. Behrouzi, "Interactive Physical Experiments in an Advanced Undergraduate Structural Dynamics Course," presented at the 2019 ASEE Annual Conference \& Exposition, Jun. 2019, Accessed: Mar. 17, 2020. [Online]. Available: https://peer.asee.org/interactive-physical-experiments-in-an-advanced-undergraduatestructural-dynamics-course.

[5] D. R. Kraemer, "Real-world Acceleration Measurements for Engineering Dynamics Course," presented at the 2017 ASEE Mid Atlantic Section Spring Conference, Apr. 2017, Accessed: Mar. 17, 2020. [Online]. Available: https://peer.asee.org/real-world-accelerationmeasurements-for-engineering-dynamics-course.

[6] S. Bevill and K. Bevill, "Smartphone-based Measurement of Acceleration: Development of a Smartphone Application for Use in an Engineering Dynamics Course," presented at the 2015 ASEE Annual Conference \& Exposition, Jun. 2015, pp. 26.1377.1-26.1377.11, Accessed: Mar. 17, 2020. [Online]. Available: https://peer.asee.org/smartphone-based-measurement-ofacceleration-development-of-a-smartphone-application-for-use-in-an-engineering-dynamicscourse. 
[7] H. Kobayashi, "Development of Motion Analysis Software for Dynamics Education," presented at the 2015 ASEE Annual Conference \& Exposition, Jun. 2015, pp. 26.536.126.536.14, Accessed: Mar. 17, 2020. [Online]. Available: https://peer.asee.org/developmentof-motion-analysis-software-for-dynamics-education.

[8] R. Vitali, N. C. Perkins, and C. J. Finelli, "Incorporating IMU Technology to Demonstrate Concepts in Undergraduate Dynamics Courses," presented at the 2018 ASEE Annual Conference \& Exposition, Jun. 2018, Accessed: Mar. 17, 2020. [Online]. Available: https://peer.asee.org/incorporating-imu-technology-to-demonstrate-concepts-inundergraduate-dynamics-courses.

[9] R. Vitali, N. C. Perkins, and C. J. Finelli, "Board 153: Continued Assessment of i-Newton for the Engaged Learning of Engineering Dynamics," presented at the 2019 ASEE Annual Conference \& Exposition, Jun. 2019, Accessed: Mar. 17, 2020. [Online]. Available: https://peer.asee.org/board-153-continued-assessment-of-i-newton-for-the-engaged-learningof-engineering-dynamics.

[10] R. Vitali, N. C. Perkins, and C. J. Finelli, "Board 155: Introduction and Assessment of iNewton for the Engaged Learning of Engineering Dynamics," presented at the 2018 ASEE Annual Conference \& Exposition, Jun. 2018, Accessed: Mar. 17, 2020. [Online]. Available: https://peer.asee.org/board-155-introduction-and-assessment-of-i-newton-for-the-engagedlearning-of-engineering-dynamics.

[11] J. Vernon, C. J. Finelli, N. C. Perkins, and B. G. Orr, "Piloting i-Newton for the Experiential Learning of Dynamics," presented at the 2015 ASEE Annual Conference \& Exposition, Jun. 2015, pp. 26.1228.1-26.1228.9, Accessed: Mar. 17, 2020. [Online]. Available: https://peer.asee.org/piloting-i-newton-for-the-experiential-learning-of-dynamics.

[12] "The PocketLab | Specifications." https://www.thepocketlab.com/specs.html (accessed Nov. 15, 2018).

[13] "Educators | PocketLab." https://www.thepocketlab.com/educators (accessed Mar. 30, 2020). 